\title{
Introducing an Online Consumer-Based Review Platform for Restaurant Hygiene
}

\author{
Wessam Atif ${ }^{1, *}$, Mohamed Farid ${ }^{2, *} \&$ Kota Kodama ${ }^{2}$ \\ ${ }^{1}$ School of Health Science, University of Manchester, UK \\ ${ }^{2}$ Graduate School of Technology Management, Ritsumeikan University, Osaka, Japan \\ Correspondence: Wessam Atif, School of Health Science, University of Manchester, Oxford Rd, Manchester M13 \\ 9PL, UK. Tel: 63-915-225-1688. E-mail: wessam.atif@postgrad.manchester.ac.uk \\ * Shared first authorship, these authors contributed equally to this work.
}

Received: November 13, 2018 Accepted: January 4, 2019 Online Published: January 23, 2019

doi:10.5539/gjhs.v11n2p118 URL: https://doi.org/10.5539/gjhs.v11n2p118

\begin{abstract}
The World Health Organization states that everyone should play a role in contributing to food hygiene. In this article, we introduce the first online consumer-based platform for restaurant hygiene reviews, a platform that may provide a transparent channel for consumers to play their role in food hygiene. While public purchase decisions may be significantly affected by online consumer reviews, currently there are no dedicated websites for consumers to add restaurant hygiene reviews (RHRs), which is an expression coined in this article. The new platform helps consumers post food hygiene reviews by answering a series of questions while visiting any restaurant, and it also gives them an option to report food hygiene violations to the authorities. This website may help future research if the data collected is analyzed to understand trends in food hygiene violations noticed by the public; we also plan to have annual awards for the best restaurant in food hygiene based on consumer reviews. The questionnaire provided will also contribute to consumer food hygiene education. This platform is expected to bring food hygiene into the context of daily life and add to pressure on the restaurant industry to follow food hygiene requirements, thereby leading to a positive impact on environmental health.
\end{abstract}

Keywords: consumer reviews, food safety, online platform, philippines, public health, restaurant hygiene

\section{Introduction}

About 420,000 people die every year because of foodborne illness and one-third of those victims are children (World Health Organization [WHO], 2015); in 2015, the WHO made it clear in its report on the global burden of foodborne diseases that everyone must play a role and contribute to improving food safety.

While the role of government agencies or food business operators may be easier to identify and to check, what about the extent of the active role consumers can play in public food safety at restaurants and similar food catering establishments? Knowing that laws and regulations alone can never provide full or absolute protection to the public (Wilcock, Pun, Khanona, \& Aung, 2004), the authors pose the following questions:

1) What do consumers currently do to put pressure on the restaurant business for better food safety and hygiene?

2) Do consumers really know the basic food hygiene requirements to look for when they are dining out?

3) What do consumers do when or if they notice a food hygiene violation in a restaurant?

4) Do customers have a direct channel to easily report violations to the authorities?

5) Is there a platform where consumers can contribute to food hygiene or food safety reviews for fellow consumers regarding the status of food hygiene in different restaurants?

Researchers may also want to collect data which can only be obtained currently from government food inspectors, for example:

1) What food hygiene violation is most commonly reported by the public?

2) What is the status of the food hygiene in a specific restaurant?

The data gathered by the government is not always adequate or representative of the real situation. We do 
understand that a food inspector may visit a restaurant only a limited number of times per year, but consumers visit more frequently and may be able to provide more data over time.

All the questions above led us to the new idea of developing the first online platform for public use specialized in restaurant hygiene reviews (RHRs) by consumers in the Philippines; we call it Eat Safe.

The platform is now active and covering most restaurants in Manila. The website is continuously undergoing improvements and being fine-tuned for better service and output. Use of the platform is completely free of charge and can be accessed via any internet-enabled device using the link www.eatsafe.ph.

\section{Solution Context}

\subsection{The Extent of Internet Use in the Philippines as of 2018}

According to the digital report issued by the Canadian social media management Hootsuite in January 2018, the population of the Philippines has reached 105.7 million. With 67 million internet users, the Philippines has reached a very high penetration rate of $63 \%$, making it the twelfth highest country in the world in terms of internet users. The number of active social media users is $100 \%$ of internet users in the Philippines. The number of unique mobile users in the Philippines has reached 61 million users for a 58\% penetration rate.

These data indicate that internet penetration in the Philippines is high, which has led to our creation of the hypothesis that the internet to give consumers a platform where their voices and opinions about food safety and hygiene can be heard.

\subsection{The Effect of Online Consumer Reviews on Public Purchasing Decisions}

By reviewing evidence on the importance of online customer reviews (OCRs) and/or electronic word of mouth (e-WOM) on consumers' purchasing decisions, we found that OCRs are now a sizable source of product and service information for customers on the internet (Kostyra, Reiner, Natter, \& Klapper, 2016), and that these reviews can have a significant effect on purchasing decisions by the public (Elwaldas, Lü, \& Ali, 2016).

One study that focused on OCRs in hotel sales concluded that better customer ratings significantly improved these sales through online platforms (Öğüt \& Taş, 2012). Electronic word of mouth is now an easily accessible source of information and may have a great effect on decisions made for online consumption (Cheung, Lee, \& Rabjohn, 2008).

\subsection{The Effectiveness of Online Posting of Restaurant Hygiene Scores by the Government}

Currently, there have been no online platforms dedicated to food hygiene reviews by consumers and, consequently, no research or evidence on the effectiveness or importance of such platforms, but we have found evidence related to similar platforms posting restaurant hygiene scores given by government agencies.

In the United States, for instance, a study was done to check the effectiveness or impact of posting "government restaurant hygiene scores" in Salt Lake County, Utah; it was found that the main critical hygiene or food safety violations were effectively decreased after the government launched the site and publicly posted restaurant food safety and hygiene scores. The study stated that compliance is believed to improve after public posting of the scores owing to consumer pressure (Waters, Porucznik, Kim, DeLegge, \& Durrant, 2013).

The role of "consumer pressure" was very enlightening for us, as we thought that such a platform would not only allow consumers to be informed about government scores for restaurants, but be fully dedicated and operated by and for consumers; we believe this may have positive effects and may help us to answer several of the questions asked at the outset in Section 1.

The EatSafe platform is thought to be the first that gives the role of food safety inspection and reviews directly to the consumer, and while platforms posting hygiene scores by enforcement agencies are good and effective, we believe that the platform proposed may help complete the picture.

\section{System Design Methodology}

\subsection{The Need for Food Hygiene Education and Knowledge}

The need for consumer education and knowledge on food safety is critical: one study in 1994 stated that with the public becoming more aware of the hazards and with decreasing uncertainty, the perception and pressure of the public on food risk control may change (Soby, Simpson, \& Ives, 1994), as the internet now has a growing popularity that makes it one of the recommended channels to communicate public food risks.

In 1995 Socket noted that many people lack basic knowledge on food safety (Sockett, 1995). We believe that our platform may contribute to educating consumers about basic food hygiene requirements. This is necessary 
according to a study on consumer food safety attitudes, behavior, and knowledge, which concluded that consumers require professional help when it comes to food safety (Wilcock et al., 2004). Providing that is one of the main functions of our website.

The same study also concluded with several recommendations that we think our platform may contribute to, including but not limited to the need for consumer food safety education, the need for awareness on the basics of safe food handling, the need to improve the public's trust on food safety information through trustworthy sources, and the need to incorporate food hygiene in the context of daily life (Wilcock et al., 2004).

The platform will also provide a direct and easy-to-use channel for consumers to report any food hygiene violations directly to the city health office, thereby making reports to authorities (if needed) easier and more accessible.

If a restaurant or food establishment is not found on the website, the consumer may easily "add a food establishment."

\subsection{Breakdown of the Platforms' Functions and Methods of Data Collection}

The main functions of the EatSafe platform are:

1) Uploading restaurant food hygiene reviews (guided by a risk-based questionnaire)

2) Uploading different comments (text reviews)

3) Uploading pictures of food hygiene violations

4) Sharing reviews to social media, such as Facebook or Twitter

5) Option to directly report the restaurant's food hygiene violations that the users are reviewing through the website to the city health office

6) Based on reviews every year, we plan to organize an award ceremony for the restaurant with best food hygiene and best contributor to reviews (the Eatsafe.ph Awards)

On the platform backend (admin view) we may also extract and mine data to help us identify patterns of different food hygiene violations over time; we would also know the total number of restaurants reported to authorities, the restaurants that were reported most frequently, which food hygiene violations were most commonly reported by the public, and other data.

This data will complement the data-gathering systems that are operated by the government or enforcement agencies and will provide the public, who may have no access to government records, with a transparent source of information about the level of food hygiene at places where they eat.

\subsection{The EatSafe Food Hygiene Questionnaire and Algorithm}

Once a user logs in and selects a restaurant to review, a risk-based questionnaire pops up to guide the user through the review process.

We believe it may not be fair, for example, to give a very negative review simply because of a single violation that may or may not be critical; that is the reason we prefer the star rating to be based on a fair and risk-based questionnaire. This means that the consumer does not actually give a specific star rating but instead answers a series of questions based on which the stars are calculated.

The questionnaire asks the user to move around the restaurant (only to where they have legal access) to check and answer a series of 13 questions covering the following universal food hygiene risk factors (Food and Agriculture Organization, 2008):
1) Cleaning and sanitizing
2) Sick food handlers
3) Holding temperatures
4) Cross-contamination
5) Personal hygiene
6) Presence of pests
7) Food allergies

A few risk factors, such as cooking temperatures, food from unsafe sources, and water quality were not included because the customer may have no access to checking them, although we may add notes encouraging consumers to 
order properly cooked food and explain the reasons.

The questions have different weightings based on our evaluation of the contribution of different factors to the risk level. Nine questions are answered by Yes or No, and four are answered on a scale from 1 to 5 or, in the case that the user has no access, she or he may answer "I cannot see" and the algorithm will simply not include that specific question in the calculation.

Based on the answers the algorithm automatically calculates a score or star rating out of five and adds it to the review. The user next has the option to write their own comments in the text, add pictures, then submit their review, or even share it to their favorite social media platform, thus bringing food hygiene reviews and information into the context of everyday life.

One major goal of our questionnaire is to help educate the consumers about what they should they be looking for with regard to food safety and hygiene; they may already be aware of various issues but the questionnaire will spark their memory and provide a more systematic approach to their review. Moreover, every question has a "see more" part to it over which a consumer may hover to get further information about the rationale behind the question, such as the reason food handlers need to cover their hair.

The other important feature is the "Report to Authorities" where the customer can simply write comments, upload photos, and send their report directly to the city health office. Once they do that, our system automatically sends the report to the e-mail address of the city health office in the city where the restaurant was registered; a control copy is then sent to our administration on the back end.

If the e-mail address of one or more city health offices is not available, it is our task to make sure we deliver the customer report to that specific city health office by other means within 24 hours.

\subsection{The EatSafe Validation Review System}

Validating the reviews to make sure that they are real and reflect actual cases is essential for the platform to succeed. Currently, the system can validate the user by allowing sign in via Facebook or Google accounts. In the case where the user signs up via e-mail, the system sends an automated validation e-mail as well. The system also requires having a valid cell phone number added to the profile at the sign-up stage to complete the process. We are now developing a validation protocol via cellphones' SMS to increase the validation level and make sure that the account holder is an actual unique user. We are also working on an integration process with a number of the latest third-party Artificial Intelligence (AI) tools that have proprietary technology in text mining and review analysis; these are able to detect any suspicious patterns. In the future, we are planning to add an extra layer of security that requires the user to take a photo of the restaurant receipt via the EatSafe mobile app (under development) to validate the review. We believe that this last layer of security represents a confirmation of the reviews' authenticity and may prevent any fake reviews.

\subsection{Platform Limitations}

The platform is mainly focused on the areas that the customer can reach and examine. The most critical place regarding food safety in the restaurant is the kitchen or preparation area. We encourage the users of the platform via the survey to tour the restaurant areas legally accessible or to view the kitchen if it is a see-through or open kitchen. However, we understand that some restaurants do not give such access. To compensate for this limitation, we are currently working with the local city health offices to upload the "inspection scores" they have given to each restaurant on our platform; by doing this, we will also be able to provide our customers with more complete and triangulated reviews from the users' and the government's perspective. A disclaimer is being added to the homepage of the website stating clearly that the results of the customer restaurant hygiene reviews on the site are only based on checking the areas of the restaurant accessible to the customer.

One more limitation we are working on is to add a channel for the restaurants to reply to the reviews uploaded by the public in case they have feedback or if they want to show improvements.

\section{Conclusions}

We believe that this platform will help increase the active role that customers can play in public food safety and hygiene, getting them more involved in demanding safe food, and adding pressure on the restaurant and catering industry to follow basic food hygiene requirements- "consumer pressure."

The awards for best restaurants and best contributors are meant to motivate interested parties and increase their involvement in the process, which we hope will improve the overall food safety and hygiene culture among consumers and restaurants. 
We believe this platform has great potential for being expanded to address public food hygiene concerns in the near future. The scalability is limitless and is planned to soon include different regions and countries outside the Philippines.

We are also aware that there are many limitations and areas of improvement for our platform; this is why we are always open to suggestions and feedback from the public and government, because only with public engagement can we have a positive impact on public health.

The support of organizations such as the Department of Health (DOH), Department of Trade and Industry (DTI), Department of Tourism, and World Health Organization in the Philippines (WHO) may also be needed to encourage the public to start using this new platform. It will be highly appreciated.

\section{Acknowledgments}

We acknowledge the support from JSPS KAKENHI Grant Numbers JP15H05183. We also appreciate all open source and database developers used in this research. There is no conflict of interest to be declared regarding this research.

\section{Competing Interests Statement}

The authors declare that there are no competing or potential conflicts of interest.

\section{References}

Cheung, C. M., Lee, M. K., \& Rabjohn, N. (2008). The impact of electronic word-of-mouth: The adoption of online opinions in online customer communities. Internet Research, 18(3), 229-247. https://doi.org/10.1108/10662240810883290

Elwaldas, A., Lü, K., \& Ali, M. (2016). Perceived derived attributes of online customer reviews. Computers in Human Behavior, 56, 306-319. https://doi.org/10.1016/j.chb.2015.11.051

Food and Agriculture Organization of the United Nations [FAO]. (2008). Risk-based food inspection manual. Rome: Food and Agriculture Organization of the United Nations.

Kostyra, D. S., Reiner, J., Natter, M., \& Klapper, D. (2016). Decomposing the effects of online customer reviews on brand, price, and product attributes. International Journal of Research in Marketing, 33(1), 11-26. https://doi.org/10.1016/j.jiresmar.2014.12.004

Ögüt, H., \& Taş, B. K. (2012). The influence of internet customer reviews on the online sales and prices in hotel industry. The Service Industries Journal, 32(2), 197-214. https://doi.org/10.1080/02642069.2010.529436

Rappler Philippines. (2015). A profile of internet users in the Philippines in partnership with Globe, 2015. Retrieved from https://www.rappler.com/brandrap/profile-internet-users-ph

Soby, B. A., Simpson, A. C. D., \& Ives, D. P. (1994). Managing food-related risks: integrating public and scientific judgements. Food Control, 5(1), 9-19. https://doi.org/10.1016/0956-7135(94)90128-7

Sockett, P. N. (1995). The epidemiology and costs of diseases of public health significance, in relation to meat and meat products. Journal of Food Safety, 15(2), 91-112. https://doi.org/10.1111/j.1745-4565.1995.tb00126.x

Waters, A. B., J., Porucznik, C. A., Kim, J., DeLegge, R., \& Durrant, L. (2013). Impact of internet posting of restaurant inspection scores on critical violations. Journal of Environmental Health, 75(10), 8-13.

Wilcock, A., Pun, M., Khanona, J., \& Aung, M. (2004). Consumer attitudes, knowledge and behaviour: A review of food safety issues. Trends in Food Science \& Technology, 15(2), 56-66. https://doi.org/10.1016/j.tifs.2003.08.004

World Health Organization [WHO]. (2015). WHO estimates of the global burden of foodborne diseases. Foodborne Disease Burden Epidemiology Reference Group.

\section{Copyrights}

Copyright for this article is retained by the author(s), with first publication rights granted to the journal.

This is an open-access article distributed under the terms and conditions of the Creative Commons Attribution license (http://creativecommons.org/licenses/by/4.0/). 